\title{
Research on the Broadband Equipment of Integrated Detection and Countermeasure System
}

\author{
Gaofeng CHENG ${ }^{1, a}$, Ting SUN ${ }^{2, b}$, Yingming $\mathrm{LU}^{3, \mathrm{c}}$, Shulai NIU ${ }^{4, d}$ \\ 1.4. Department of Control Engineering, Academy of Armored Forces Engineering, Beijing, China \\ ${ }^{2}$ Beijing Special Vehicle Academy, Beijing, 100072, China \\ 3.Department of Training, Academy of Armored Forces Engineering, Beijing, China

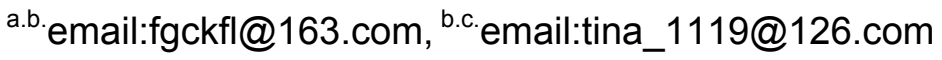

Key words: broadband, transmitter, receiver, Jammer

\begin{abstract}
Through the analysis of the radar and jammer transmitter and the antenna system, a shared broadband transmitter and antenna design was proposed, which can transmit signals and interference detection in $8 \sim 16 \mathrm{GHz}$. Through the analysis of the radar receiver and jammer, a design of integrated receiver based on channelized receiver was proposed, it can not only detect the enemy radiation signal such as the frequency, but also receive and process own radar signals.
\end{abstract}

\section{Introduction}

Radar and electronic countermeasure is an important part of information war, and is the backbone elements to capture the information dominance in the war of the future. The integration of radar and electronic countermeasure meeting the needs of operational command in the future is an inevitable trend in the development of military electronic warfare systems. Exploration and integration of radar and confrontation has great significance in improving the high-tech ability of our army and winning future high-tech war.

As we all know, in order to obtain target information, the radar must transmit high power electromagnetic energy onto the target at first. Due to electromagnetic scattering of the target, the electromagnetic energy will produce a corresponding modulation and scattering, part of which is received by the radar to demodulate the target information based on the relative relationship between the modulated transmitted and received signals[1]. The reconnaissance part of jammer receiving the radar transmit signal (containing a different radar signature) to identify the radiation source and guide the jammer cast radar interference in the airspace, the frequency-domain and time-domain. The function components of radar and jammer have many similarities, such as they both have the transmitter, the receiver, the antenna, the signal processing system, etc. But their functions and the performance requirements of each part are different.

\section{BROADBAND SHARING TRANSMITTER AND ANTENNA SYSTEM}

\section{A. Transmitter and antenna system difference between radar and jammer}

Working frequency and bandwidth is the most significant difference of radar and jammer, which is embodied by transmitter and antenna system on hardware equipment. In general, for the characteristics of microwave devices, high-power transmitting tubes, whose working bandwidth is very narrow, is mainly used in radar transmitter. And broadband transmitting tubes with lower power is mainly used in jammer. For radar's accurate positioning, antenna of high gain and narrow beam is required in order to reduce range ambiguity and pulse wave transmitted by radar. But for a jammer in order to achieve the tactical objective of area cover, relatively wide beam and adaptability to a variety of radar polarization is needed. To jam enemy radar, jammers transmitter continuous wave, whereas pulse signal resembling radar signal is also transmitter for delusion. Incompatibility makes integration equipment cannot simply share transmitter system. Therefore, the problem of sharing transmitter antenna system is the first step of researches on the integration of equipment. 


\section{B. Working frequency band selection}

Every transmitter has a certain frequency range. Currently there is no transmitter witch can work in all frequency. Therefore transmitter with wider working frequency range, is called broadband compared with general radar.

The airborne radar main work in the $8 \sim 18 \mathrm{Ghz}$. Airborne weapons and airborne radar guidance is a killer for the common ground radar, and is the first weapon of the enemy attack. So in order to improve the survival probability of radar and weaken the enemy attack ability, the main interference target for integration equipment shall be locked for airborne radar.

From the above analysis we can see that in order to improve the utilization of frequency resource, the existence ability of radar, the ability in influence of the enemy attack, the integration equipment working frequency should be selected in the $8 \sim 18 \mathrm{Ghz}$.

\section{Sharing transmitter and antenna system design}

Sharing system need to be able to transmit interference signal and radar signal. However there is no broadband transmitting tube compatible with both continuous wave and pulse wave. Sharing system design is shown in figure 1.

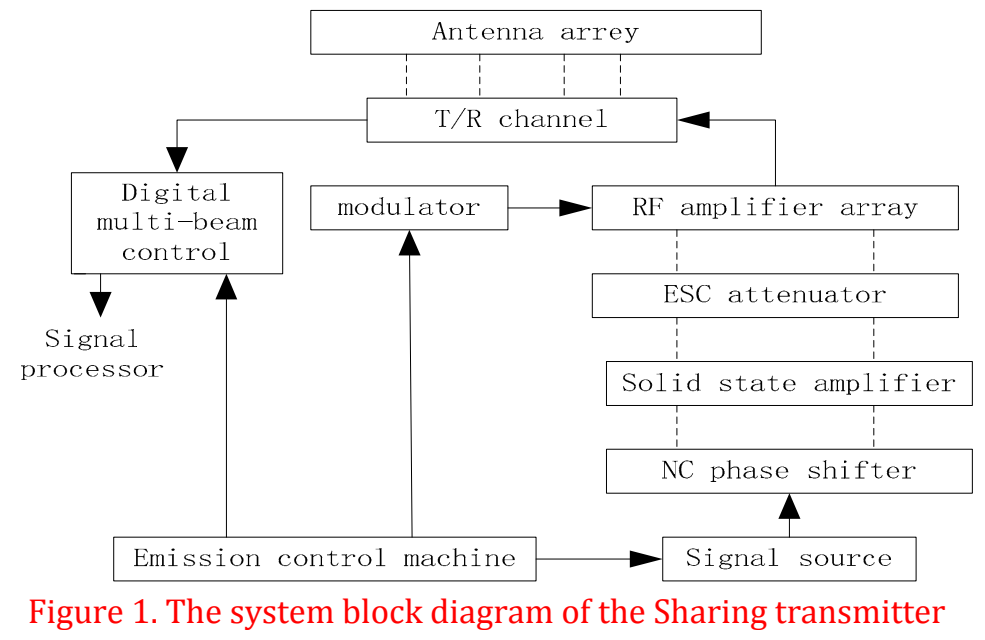

The method of to solve technical problems of sharing is described below. Emission control is composed of a high speed computer and control circuit. According to the operational requirements, select a transmit frequency. The frequency code is transmitted to the signal source generator to generate signal of specific frequency, used in microwave signal interference or detecting, and passed to the digital phase shifter; emission control also transmits the phase shift control code to the digital phase shifter. Because of the wide frequency, phase shifter and amplitude frequency characteristic inconsistency and line loss, phase shifter output can make the signal amplitude inconsistent. So the amplitude control code from electrically controlled attenuator for consistency of the amplitude modulation is needed. Microwave signal after amplitude and phase modulation, amplified by traveling wave tube power amplifier with the bandwidth of $6.5 \sim 18 \mathrm{G}$, and is sent to the antenna array. The transmitted signal integrates power in space. Digital controlled multi beam forming device uses digital processing to deal with antenna receiving signal, which is according to the different beam needed of radar and jammer control. If using the detection and jammer signal in ref [2] [3], it can detect and jam at the same time. We use digital adaptive multi beam control technology, which makes the transmitted beam can adapt to low amplitude lobe, also can improve the ability of measuring target angle and multi-target detection effect, or using multi beam intensive interference for the target interference needed region.

Because of the character of phase frequency and the amplitude frequency of phase shifter, their amplitude and phase will change, when different frequency microwave signal passing the phase shifter. It will make the beam pointing deviation from the specified direction. The phase shifter output must be corrected. As the phase shifter character is complicated for different frequency phase, artificial method to carry out the correction will be very complex and difficult to complete, and the phase compensation cannot achieve effective phase correction for all frequencies. Supercomputing 
ability can be used by the method of automatic program of phase shifter phase shift to adjust the amount. But it is only the phase shift correction. Under the condition of constant the direction of the beam, amplitude deviation will make equivalent radiated power decline in the direction, reducing the efficiency of synthesis. Only amplitude and phase correction of microwave channel at the same time, the desired consistency of amplitude and phase of signal can be achieved.

Ultra wideband high power traveling wave tube is one of the key devices in electronic warfare equipment. A jammer can jam several octave band, and increasing power can effectively strengthen the interference effect. The power is enough can be used to detect distant targets. It is also a key device integration facility location. Ultra wideband high power traveling wave tube is difficult to develop, which is the main microwave tube type under development at home and abroad. The tube can do $6.5 \sim 18 \mathrm{GHz}$ with the output power more than $200 \mathrm{~W}$ abroad. The domestic production of 7.5 $\sim 16.5 \mathrm{GHz}$, the output power is greater than $200 \mathrm{~W}$ tubes, and development of $6.5 \sim 18 \mathrm{GHz}$, the output power is greater than $180 \mathrm{~W}$ pipe.

For the integration equipment to realize the detection function, generally the existing fuzzy of distance in continuous wave signal, and the pulse signal has more effective ranging capability. But the interference from pulse wave cannot be implemented in full time domain continuous interference to enemy radar, because the pulse interval period cause the formation of interference loopholes zone. When transmitting pulse signals, with phase controlled array system, and the control system by using multi beam, we can use beam relay method to control different transmitting tube, to make the beam in time continuous interfere target beam, when the power needed is satisfied. It makes the pulse interference signal, forming a continuous wave interference effect in the enemy's radar receiver.

\section{BROADBAND SHARING RECEIVER}

A. Difference between radar and jammer receiver

Radar receiver and jammer receiver have different functions due to their different operational missions. The mission of radar receiver is to detect the parameter information of target echo (distance, orientation, speed, etc.) from a variety of noise and clutter. The radar's received frequency can be pre-tuned, so it is easy to use matched filter processing. To achieve the purpose of the reconnaissance, the mission of jammer is receiving any signals of interest in a wide frequency range electronic warfare, so the bandwidth of its former and back stage filters vary widely.

\section{B. Broadband sharing receiver design}

In order to achieve the integration of the receiver, multiple channelsof the channelized receiver are used to achieve full-band reconnaissance function, and one of them is selected as the private channel of a radar receiver.Figure 2 shows the system design.

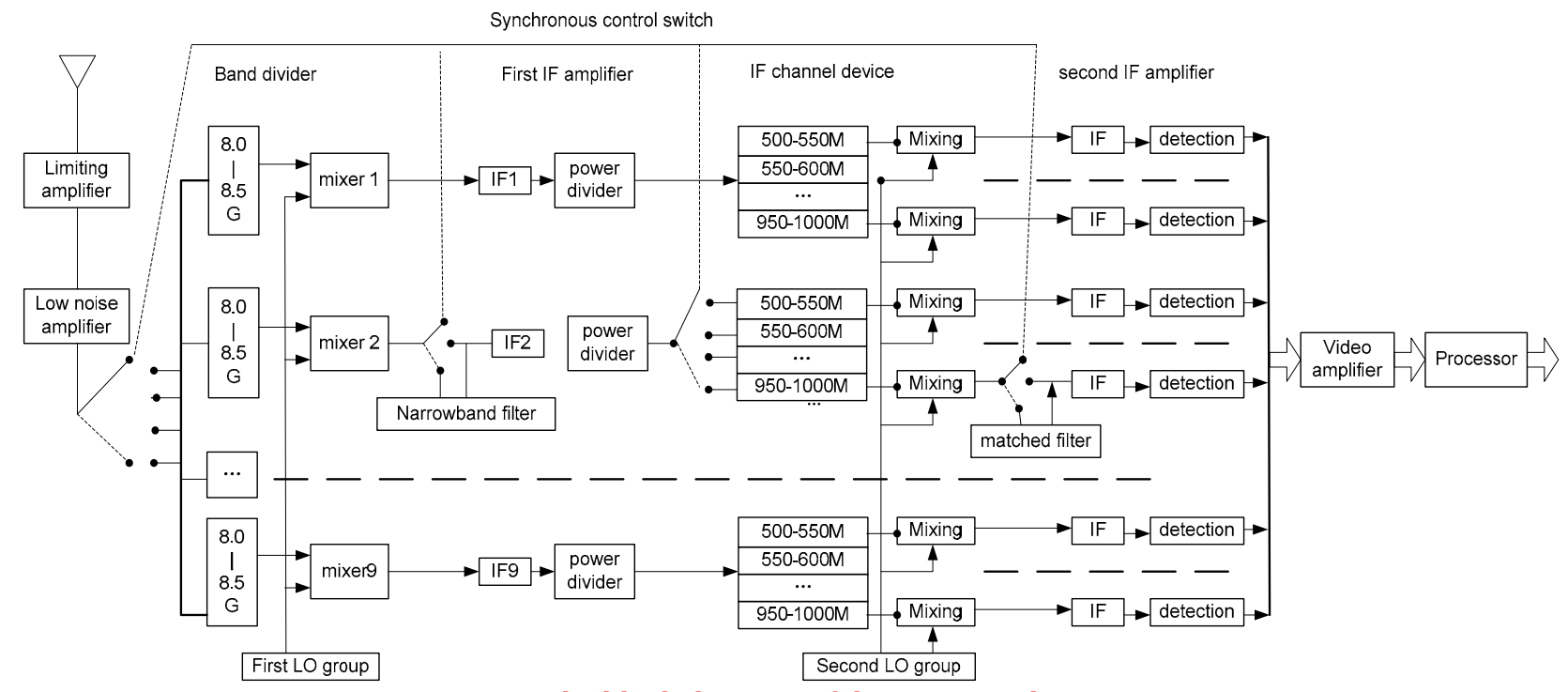

Figure 2.The block diagram of the integrated receiver 
The input RF bands (8-12.5G) are shunted by 9 adjacent parallel filters with 500M bandwidth in this scheme. Frequency channel's 9 outputs then respectively down converted to a shared intermediate frequency, whose center frequency is $750 \mathrm{M}$. The intermediate frequency channel can be further shunted by 10 adjacent parallel filters with $50 \mathrm{M}$ bandwidth. Finally it is respectively converted to $30 \mathrm{M} \mathrm{IF}$, and be sent to video and processor after detection.

The integrated receiver works as follows: The synchronized switch control the timely sharing work of radar receiver and reconnaissance receiver. While the radar receiver works, the synchronized switch will separately connect the narrowband filter and the matched filter in front and behind the detector, completing the detecting and receiving process of radar. There is a blocked circuit added in front of the receiver for blocking signals transmitted by nearby friendly radar, which will reduce the difficulty of subsequent signal processing. When receiving a continuous wave signal, the PIN switch will alarm and send a pulse to modulate the continuous wave for processing.

\section{Summary}

The broadband equipment of integrated detection and countermeasure system is a frontier problem of equipment research. It involves system design development, phased array technology, receiver and process module of shared signals, multi-band radar, complex signal generation, multi-band digital frequency source, intelligent research, etc. Currently, research in this respect is very little at home. This paper presents some exploratory research on broadband transmitter, antenna systems and receivers, which have a reference role for the research on the broadband equipment of integrated detection and countermeasure system.

\section{References}

[1] Zhao Guoqing. Principle of Radar Countermeasure. Shaanxi: Xidian University Press, 1999.

[2] Wu Hong, Xu Ziwen, Wu wen. Analysis of Hybrid Noise Modulated Radar Signal Based on Discrete Pulse Position. Journal of Air Force Radar Academy, 2002, 16(4):22-24.

[3] Xu Cuichun, Chen Tianlin. Conception and Signal Design Ideology of Integration of Interference and Radar Positioning. New concepts, theories and Techniques of Electronic Warfare.2001.

[4] D. Falconer, S. L. Ariyavisitakul, A. Benyamin-Seeyar, and B. Eldson,"Frequency domain equalization for single-carrier broadband wireless systems," IEEE Commua. Mag, vol. 40, no. 4, pp. 58-66, 2002.

[5] J. Karjalainen and T. Matsumoto, "On the convergence property of an MMSE multiuser MIMO turbo detector with uplink precoding," pre-sented at the IEEE Int. Conf. Commun., Beijing, China, May 19-23,2008.

[6] 3rd Generation Partnership Project (3GPP); Technical SpecificationGroup Radio Access Network Evolved Universal Terrestrial Radio Ac-cess -UTRA, "Physical Channels and Modulation," 3GPP TS 36.211,ver. 12.1.0 (Release 121 Tech. Ren,2014.

[7] T. Saeki, 5. Sato, M. Kurokawa, A. Moto, M. Suzuki, K. Tanaka,K. Tanaka, N. Ikama, and Y. Fujimura, "100 GbiVs compact transmitter module integrated with optical multiplexer;' in Proc. IEEE Photon Conf, 2013, pp. 307-308.

[8] S. Seguin, "Detection of low cost radio frequency recelve rs based on their unintended electromagnetic emissions and an active stimulation;Ph.D dissertation, Dept. Electr. Comput. Eng., Missouri Univ. SciTechnnl.. Rnlla. MD. IISA,2009.

[9] RichardRnsic,J. A Scheer, and W. A. Holm, Principles of Modern West Perth, Australia: SciTech Publishing, 2010.

[10]J. D. Shirman, Computer Simulation of Aerial Target Radar Scat-tering Recognition, Detection, Traclahg. Norwood, MA, USA: Artech Honse,2002. 